\title{
AN EXTENSION OF THE LENNARD-JONES AND DEVONSHIRE MODEL TO LIQUID CRYSTALLINE PHASES
}

\author{
P. J. PHOTINOS
}

Department of Physics

\section{A. SAUPE}

Liquid Crystal Institute and Department of Physics, Kent State University, Kent, Ohio 44242 U.S.A.

\begin{abstract}
Résumé. - Une théorie à pression constante sur la fusion est présentée. Cette théorie prédit une phase caractérisée par un ordre translationnel dans une dimension. Des théories semblables ont été déjà développées par un réseau incompressible. Ce modèle, qui est une extension de celui présenté par Lennard-Jones et Devonshire, utilise un double réseau associé à deux réseaux conjugués, ce qui donne deux modes de fusion.

Le premier correspond à la fusion dans les plans, le second à la fusion smectique. La distinction entre les deux modes est attribuée à l'ordre orientationnel à large distance. Le dernier n'est pas inclus directement dans le modèle statistique. Un terme quadratique représentant l'ordre orientationnel est ajouté à l'expression de l'énergie libre.

Les valeurs du paramètre d'ordre nématique sont obtenues à partir d'une équation de self-consistance.

En utilisant les facteurs de compressibilité tabulés on obtient les isothermes pour discuter les transitions de phase cristal-nématique et smectique-nématique.
\end{abstract}

\begin{abstract}
A constant pressure theory of melting is presented which predicts a phase characterized by translational order in one direction. Similar theories have been already developed for an incompressible lattice. The present model, which is an extension of the one developed by LennardJones and Devonshire, uses a twin lattice, along with the conjugate lattices, and hence allows for two modes of melting. One mode corresponds to the melting within the planes, the second represents the smectic melting. The distinction between the two modes is attributed to the long-range orientational order. The latter is not included directly in the statistical model; instead, a quadratic term representing the orientational order is added to the expression for the free energy. The values of the nematic order parameter are derived from a separate self-consistency equation. Using tabulated compressibility factors we obtain the isotherms, which are used to discuss the crystal to smectic and smectic to nematic phase transitions.
\end{abstract}

1. Introduction. - A lattice-model [1] description of the liquid state was first presented by Lennard Jones and Devonshire [2]. According to this description, the $N$ molecules are distributed on two interpenetrating lattices, $\alpha$ and $\beta$, having $N$ sites each. The arrangement where most of the molecules lie on the sites of one lattice, corresponds to the solid, while the liquid corresponds to disordered equidistribution of molecules between the two lattices. In other words, melting is regarded as an order-disorder transformation. It is assumed that the forces involved are additive, and no multiple occupation is permitted. To simplify the mathematical treatment, one can neglect all short-range order, apart from what follows from the long-range order, and apply the Bragg and Williams method [3].
Pople and Karasz [4] extended the Lennard-Jones and Devonshire model to account for both positional and orientational melting, by allowing each molecule to take up one of two orientations on any site. The two transitions are separate for small rotational potential barriers, and coalesce for large barriers. Chandrasekhar et al. [5] modified the above mentioned work, to discuss the solid to nematic, and nematic to isotropic transitions. In the present work, we will attempt an extension of the lattice model to liquid crystalline phases, including the smectic arrangement.

2. Theoretical. - Here we will assume two uncoupled lattices, $\alpha_{1}$ and $\alpha_{2}$, along with their conjugate lattices $\beta_{1}$ and $\beta_{2}$. Each molecule can occupy one site on lattice $1\left(\alpha_{1}\right.$ or $\left.\beta_{1}\right)$ and one on lattice $2\left(\alpha_{2}\right.$ or $\left.\beta_{2}\right)$. By 
introducing these two lattices we are in effect introducing two modes of melting. Lattice 1 can be pictured to describe the xy-plane, and lattice 2 the third direction. Thus long-range order in lattice 2 along with disorder in lattice 1 corresponds to a layered structure, long-range order in both corresponds to a crystal, etc. The distinction between the two lattices should be introduced through the orientational long-range order (if any). We shall not attempt at this point to tie the statistical model to any rigorous geometrical picture. Thus, the lattices are introduced here mainly to provide the necessary energy states. The forces are assumed additive. Let $W_{i}$ be the energy corresponding to a nearest neighbour $\alpha_{i} \beta_{i}$ interaction, $z_{i}$ the number of $\beta_{i}$-sites adjacent to each $\alpha_{i}$-site, and $Q_{i}$ the fraction of occupied $\alpha_{i}$-sites $(i=1,2)$. Then in the BraggWilliams approximation one can write

$$
\ln \left(\frac{Q_{i}}{1-Q_{i}}\right)=\frac{z_{i} W_{i}}{2 k T}\left(2 Q_{i}-1\right)
$$

and as usual, $Q_{i}=1 / 2$ is always a solution, corresponding to the disordered state, while for sufficiently low temperatures, solutions with $Q_{i} \neq 1 / 2$ exist, corresponding to spatial long-range order.

The Helmholtz free energy consists of two parts; one contribution from the completely ordered system, $A^{\prime}$, and one due to the deviation from perfect order, $A^{\prime \prime}$ [4]

$$
\begin{array}{r}
\frac{A^{\prime \prime}}{N K T}=\sum_{i=1}^{2} 2 Q_{i} \ln Q_{i}+2\left(1-Q_{i}\right) \ln \left(1-Q_{i}\right)+ \\
\frac{z_{i} W_{i}}{k T} Q_{i}\left(1-Q_{i}\right)
\end{array}
$$

and the resulting contribution, $p^{\prime \prime}$, to the total pressure $P$, is

$$
p^{\prime \prime}=-\left(\frac{\partial A^{\prime \prime}}{\partial V}\right)_{T}=-N \sum_{i=1}^{2} z_{i} Q_{i}\left(1-Q_{i}\right)\left(\frac{\partial W_{i}}{\partial V}\right)_{T} .
$$

For the first contribution we shall use the results of Wentorf et al. [6] and following these authors $V_{0}$ will denote the volume of the assembly when the particles occupy a face-centered lattice, with nearest neighbor distances equal to the distance minimizing the 6-12 potential. This procedure becomes questionable when applied to molecules of interest to liquid crystalline phases, it seems worthwhile nevertheless to adopt this procedure as a first step.

In order to account for the orientational order, we shall include an additional term in the free energy. This contribution will be conveniently expressed as

$$
\begin{aligned}
\frac{A^{\prime \prime \prime}}{N k T}=\frac{1}{2}\left(1+\eta^{2}\right) & \frac{W_{\theta}}{k T}-\ln \int_{0}^{1} \mathrm{~d} \cos \theta \times \\
& \exp \times\left\{\eta W_{\theta} P_{2}(\cos \theta) / k T\right\}
\end{aligned}
$$

where $P_{2}(\cos \theta)$ is the Legendre polynomial of second order and

$$
\eta=\frac{\int_{0}^{1} \mathrm{~d} \cos \theta P_{2}(\cos \theta) \exp \left\{\eta W_{\theta} P_{2}(\cos \theta) / k T\right\}}{\int_{0}^{1} \mathrm{~d} \cos \theta \exp \left\{\eta W_{\theta} P_{2}(\cos \theta) / k T\right\}}
$$

i.e., the self consistency equation for the nematic phase [7]. Next, we have to specify the variation of $W_{1}, W_{2}$ and $W_{\theta}$ with volume. Here we will assume

$$
\begin{aligned}
& W_{1}=W_{01}\left(V_{0} / V\right)^{4} \\
& W_{2}=W_{02}\left(V_{0} / V\right)^{4} \\
& W_{\theta}=W_{0 \theta}\left(V_{0} / V\right)^{3}
\end{aligned}
$$

We shall further assume that

$$
W_{02}=(1+l \eta) W_{01}
$$

which implies that in the absence of orientational long-range order the two translational modes coalesce.

Using Eqs. (3) and (4), we can write

$$
\begin{gathered}
\frac{P V_{0}}{N k T}=\frac{p^{\prime} V_{0}}{N k T}+4\left(V_{0} / V\right)^{5} \sum_{i=1}^{2} \frac{z_{i} W_{0 i}}{k T} Q_{i}\left(1-Q_{i}\right)+ \\
\frac{3}{2}\left(V_{0} / V\right)^{4} \frac{W_{0 \theta}}{k T}\left(1-\eta^{2}\right)
\end{gathered}
$$

The term $p^{\prime} V_{0} / N k T$ is the so-called compressibility factor, and can be obtained from tables [6] as a function of $V / V_{0}$ and $k T / \varepsilon_{0}$, where $\varepsilon_{0}$ is the maximum energy of the assumed 6-12 interaction. The quantities $Q_{i}$, and $\eta$ in Eq. (8) are obtained from Eq. (1) and Eq. (5) respectively.

3. Numerical results and discussion. - Introducing the notation

$$
\begin{aligned}
a & =\frac{z_{1} W_{01}}{\varepsilon_{0}} \\
b & =\frac{z_{2}}{z_{1}} \\
m & =\frac{W_{0 \theta}}{z_{1} W_{01}} \\
v & =V / V_{0} .
\end{aligned}
$$

Eq. (8) can be recast in the form

$$
\begin{gathered}
\frac{P V_{0}}{N k T}=\frac{p^{\prime} V_{0}}{N k T}+4 \frac{a \varepsilon_{0}}{k T v^{5}}\left\{Q_{1}\left(1-Q_{1}\right)+b(1+l \eta) \times\right. \\
\left.Q_{2}\left(1-Q_{2}\right)+\frac{3}{8} m\left(1-\eta^{2}\right)\right\} .
\end{gathered}
$$

Using the above equation, along with Eqs. (1) and (5), and the tabulated values of the compressibility factor 
(extrapolating when necessary), we can plot the isotherms. The numerical results indicate that the model predicts a number of stable phases. In the framework of the present model, a stable phase with $\left(Q_{1} \neq 1 / 2\right.$; $Q_{2} \neq 1 / 2 ; \eta \neq 0$ ) describes a crystal with orientational long-range order; $\left(Q_{1}=1 / 2 ; Q_{2} \neq 1 / 2\right.$; $\eta \neq 0)$ the smectic phase; $\left(Q_{1}=Q_{2}=1 / 2 ; \eta \neq 0\right)$ the nematic phase; and $\left(Q_{1}=Q_{2}=1 / 2 ; \eta=0\right)$ corresponds to the isotropic liquid. The isotherms for the set of parameters $(a=3 ; b=1 ; l=1 ; m=1)$ for $k T / \varepsilon_{0}=0.4$ and 0.42 are plotted in figure 1 . The

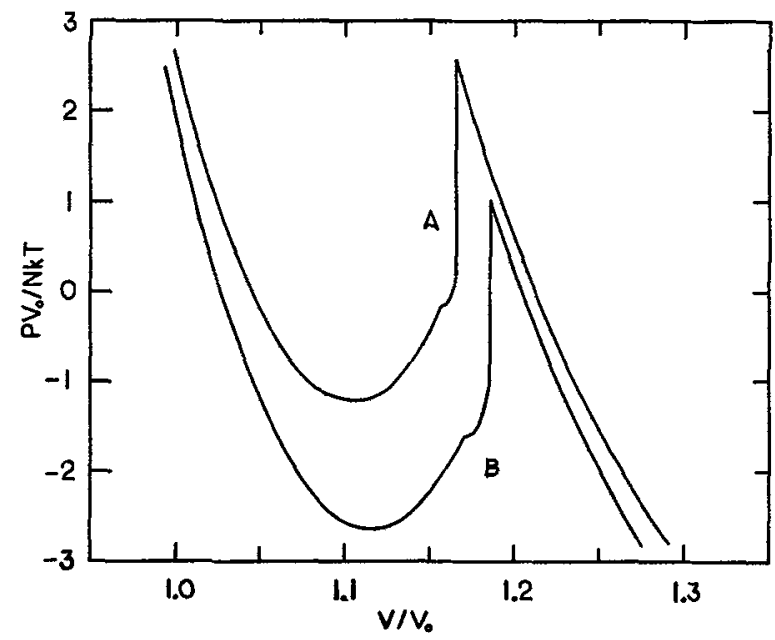

FIG. 1. - Theoretical isotherms for the set of parameters $(a=3$; $b=1 ; l=1 ; m=1$ ) for (A) $k T / \varepsilon_{0}=0.42$, and (B) $k T / \varepsilon_{0}=0.4$.

curves were plotted for reduced-volume intervals equal to 0.002 . The jump marks the disappearance of the orientational order. The disappearance of the spatial long-range order in the xy-plane (i.e. $Q_{1}$ ) is marked by the kink in the curve. By requiring that the areas above and below the zero-pressure line be equal, we have determined that the model predicts a firstorder transition from a crystal with orientational order, to an isotropic liquid at $k T / \varepsilon_{0}=0.4237$. The isotherms for the set of parameters $(a=3 ; b=1$; $l=1, m=2)$ for $k T / \varepsilon_{0}=0.48$ and 0.52 are plotted in

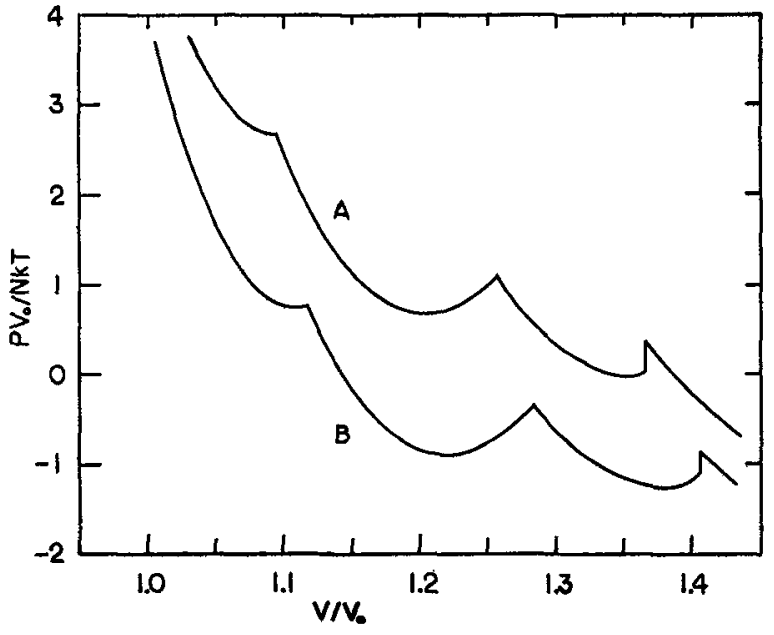

FIG. 2, - Theoretical isotherms for the set of parameters $(a=3$; $b=1 ; l=1 ; m=2)$ for (A) $k T / \varepsilon_{0}=0.52$, and (B) $k T / \varepsilon_{0}=0.48$.

figure 2. Here we observe two separate kinks. Each kink marks the disappearance of one form of long range order $\left(Q_{1}\right.$, and $Q_{2}$, in order of increasing volume). The jump is attributed to the disappearance of the orientational order. Again, by equating the areas above and below the zero-pressure line we have determined that the model predicts three firstorder transitions namely, a transition from the crystalline state to the smectic phase at

$$
k T / \varepsilon_{0}=0.4652,
$$

followed by a transition to the nematic phase at $k T / \varepsilon_{0}=0.4985$, and finally a transition from the nematic to the isotropic liquid at $k T / \varepsilon_{0}=0.518$. Similar results were obtained using a few different sets of parameters, although the search is by no means complete. It should be pointed out that the relative strengths of the transitions predicted here are not satisfactory. Before engaging in a more detailed parameter study, it seems necessary to improve on a number of points, such as the compressibility factor, the isotherm equation, and the meaningful range of parameters.

\section{References}

[1] Barker, J. A., Lattice Theories of the Liquid State, The International Encyclopedia of Physical Chemistry and Chemical Physics, Topic 10, Rowlinson, J. S., ed (MacMillan, New York) 1963. Vol. 1.

[2] Lennard-Jones, J. E. and Devonshire, A. F., Proc. Roy. Soc. A 169 (1939) 317; Proc. Roy. Soc. A 170 (1939) 464.

[3] Bragg, W. L. and Williams, E. J., Proc. Roy. Soc. A 145 (1934) 699.
[4] Pople, J. A. and Karasz, F. E., J. Phys. Chem. Solids 18 (1961) 28.

[5] Chandrasekhar, S., Shashidhar, R. and Tara, N., Mol. Cryst. Liq. Cryst. 10 (1970) 337.

[6] Wentorf, R. H. Jr., BueHLer, R. J., Hirschfelder, J. O. and Curtiss, C. F., J. Chem. Phys. 18 (1950) 1484.

[7] Marer, W. and SAUPE, A., Z. Naturforsch, 159 (1960) 287. 